\title{
OLIVIA CARPI
}

\section{ÉLITES CITADINES ET SÉDITION EN FRANCE À L'ÉPOQUE DES TROUBLES DE RELIGION}

S'agissant de la France d'Ancien Régime, quand on parle de révoltes, on pense immédiatement aux fameuses "fureurs paysannes « du XVII ${ }^{e}$ siècle, symptomatiques d'une "permanence du refus" de la part de "masses dépourvues de parole officielle «' On pense aussi aux révoltes nobiliaires qui ont émaillé l'histoire du royaume de la fin du Moyen Âge à la Fronde et qu'on n'oserait plus guère qualifier d'»imbécile turbulence « du fait de la mise en lumière de l'idéal politique et social qui les sous-tendait ${ }^{2}$. Les villes, en revanche, ne s'imposent pas d'emblée comme les protagonistes autonomes de révoltes de grande ampleur dont elles apparaissent plutôt comme les victimes ou les complices, offrant selon les cas un refuge ou un exutoire aux insurgés ${ }^{3}$.

Il est vrai que, dans les sources contemporaines, on ne fait pas toujours la distinction entre les "tumultes«, »émotions« ou »émeutes«, mouvements populaires, spontanés et très circonscrits, "sans projet politique, sans autre leaders que circonstanciels" et les authentiques "séditions« qui sont une révolte délibérée, ouverte et prolongée contre l'autorité publique, de la part de factions ou de partis, recrutant dans les couches supérieures de la société citadine mais dotés d'une forte capacité d'entraînement car »portés par une revendication unanimiste ${ }^{4}$.

\footnotetext{
${ }^{1}$ Boris PORCHEV, Les soulèvernents populaires en France de 1623 à 1648, Paris 1963 (traduction française); Roland MOUSNIER, Fureurs paysannes. Les paysans dans les révoltes au XVII $^{e}$ siècle (France - Russie - Chine), Paris 1967; Yves-Marie BERCÉ, Croquants et Nupieds. Les soulèvements paysans en France du XVI ${ }^{e}$ au XIX siècle, Paris 1974; Madeleine FoISI, La révolte des Nu-pieds et les révoltes normandes de 1639, Paris 1970; René PILLORGET, Les mouvements insurrectionnels en Provence entre 1596 et 1715, Paris 1975; Jean NiCOLAS, La rébellion française. Mouvements populaires et conscience sociale, 1661-1789, Paris 2002.

${ }^{2}$ Arlette JOUANNA, Le devoir de révolte. La noblesse française et la gestation de l'État moderne (1559-1661), Paris 1989, p. 8-10.

${ }^{3}$ Yves-Marie BERCÉ, Révoltes et révolutions dans l'Europe moderne (XVI $-\mathrm{XVIII}^{\mathrm{e}}$ siècles), Paris 1980.

${ }^{4}$ Lucien BÉlY (dir.), Dictionnaire de l'Ancien Régime, Paris 1996, p. 480-481; William H. BEIK, Urban Factions and the Social Order during the Minority of Louis XIV, dans: French Historical Studies 15/1 (1987), p. 62-63; Peter BLICKLE (dir.), Résistance, représentation et communauté, Paris 1998, p. 87.
} 
Parmi ces séditions urbaines de grande envergure, la Ligue occupe une place importante, quoique longtemps sous-estimée, alors qu'elle présente bien toutes les caractéristiques d'une authentique révolte, du moins si on la définit comme un sépisode insurrectionnel de surgissement de la violence collective dans le jeu politique, dérangeant le cours pacifique des institutions et se traduisant par l'exclusion des règles ordinaires, le choix d'une rupture et le déchaînement des armes $^{5}$. Cela tient, d'une part, aux préjugés tenaces qui se sont attachés à un mouvement considéré comme »antinational, antilibéral et ultramontain«, de l'autre, à une certaine focalisation des historiens sur »la Ligue des princes« ou sur le cas parisien, à cause notamment des analogies qu'ils y voyaient avec la Révolution française et, enfin, au faible développement de la recherche historique sur les villes du $\mathrm{XVI}^{\mathrm{e}}$ siècle, pénalisée par des sources inexistantes ou fragmentaires ${ }^{6}$.

Pourtant, l'adhésion d'un grand nombre de villes françaises à la Sainte Union, dès le printemps 1588 pour certaines et fin 1588-début 1589 , pour la plupart, entraîne une rupture ouverte avec le monarque dont elles ne reconnaissent plus l'autorité, allant même jusqu'à s'arroger certaines de ses prérogatives. Les dirigeants de ces cités font également publier des manifestes justificatifs de la rébellion qui sont autant de déclarations de guerre au souverain "tyrannique« ou "hérétique« et un vibrant appel aux villes voisines et à la noblesse locale à se dresser également contre les royaux, présentés comme leurs ennemis. Grâce à une intense mobilisation militaire et financière et à une surveillance étroite de la population, destinée à éviter toute trahison ou tout excès susceptibles de menacer l'ordre établi, les villes ligueuses parviennent ainsi à tenir tête au roi jusqu'en 1594, voire 1598, nombre d'entre elles ayant d'ailleurs cédé par la négociation et non par la force.

Certes, rien de tout cela n'aurait été possible sans une forte participation populaire, observable lors d'évènements clefs comme les barricades parisiennes du 12 mai 1588 ou après l'assassinat des Guise, le 23 décembre 1588, mais aussi tout au long de l'insurrection pendant laquelle les populations concernées ont fait face à une insécurité chronique, à la pression fiscale, aux difficultés frumentaires et aux attaques pesteuses ${ }^{7}$. Néanmoins, si le "peuple« a

${ }^{5}$ BERCÉ, Révoltes et révolutions (voir n. 3), p. 8.

6 Jean-Marie CONSTANT, La Ligue, Paris 1996, p. 468; Élie BARNAVI, Robert DESCIMON, La Sainte Ligue, le juge et la potence, Paris 1985, préface de Denis RICHET, p. 6-7; Emmanuel LeroY-LADURIE (dir.), Histoire de la France urbaine, t. III, Paris 1981; Guy SAuPIN, Les villes en France à l'époque moderne, Paris 2002; Philippe GUIGNET, Les sociétés urbaines dans la France moderne, Paris 2006.

${ }^{7}$ Henry HeLleR, Iron and Blood. Civil Wars in Sixteenth Century France, Londres 1991, p. 107; Stuart CAROLL, The Revolt of Paris, 1588: Aristocratic Insurgency and the Mobilization of Popular Support, dans: French Historical Studies 23/2 (2000), p. 301-337; Alexander WILKINSON, Homicides royaux: the Assassination of the Duc and Cardinal de Guise and the Radicalization of French Public Opinion, dans: French History 18/2 (2004), p. 129-153. 
parfois pris l'initiative du soulèvement ligueur ou bien encore celle du ralliement à Henri IV, ce n'est pas lui qui, sur le long terme, a assuré la conduite de la révolte, mais les membres de l'élite bourgeoise qui ont su mettre à profit à cette occasion leurs compétences professionnelles et leur culture, leur expérience du pouvoir, leurs moyens matériels et leurs relations interpersonnelles ${ }^{8}$. En fait, indépendamment des accusations volontairement dégradantes de ses adversaires, la Ligue des villes se présente bien comme "une révolution des notables ${ }^{9}$. Toutefois, si on comprend aisément que les plus humbles aient eu de bonnes raisons de se révolter, avec cette arrière-pensée qui veut que "ceux d'en bas recèleraient une sorte de vérité sociale qui en dirait plus sur le passé que les élites ou les porte-parole du pouvoir«, qu'en est-il de "ceux qui tenaient le premier rang dans la ville ${ }^{10}{ }^{10}$ ?

Bien sûr, loin de n'être que le simple paravent de visées plus triviales, le mobile religieux s'avère prédominant chez les bourgeois ligueurs dont le "refus prioritaire" est celui de l'hérétique et de la diversité religieuse ${ }^{11}$. Cependant, cela n'est nullement incompatible avec la poursuite d'une action politique et sociale, les contemporains eux-mêmes ne séparant pas ces différents aspects $^{12}$. De ce point de vue, la Ligue apparaîtrait alors comme la résultante des ambitions rivales de bourgeois enrichis et instruits, avides d'ascension et de considération sociales, se traduisant notamment par un accès aux charges publiques, royales ou municipales, source d'honneur et de pouvoir.

C'est là une approche féconde, qui a largement dominé l'historiographie de la Ligue urbaine et dont il importe de souligner les acquis. Cela étant, elle n'est pas sans limites. Non seulement elle a tendance à minimiser les aspects religieux, ravalés au rang de »sous-produits de forces sociales plus profondes « mais faut-il, de surcroît, ramener nécessairement le choix partisan des individus à leur statut socioprofessionnel ou aux relations verticales et horizontales dans lesquelles ils s'inséraient ${ }^{13}$ ? Car on se doute bien que les bourgeois li-

${ }^{8}$ Robert DESCLMON, La Ligue à Paris (1585-1594): une révision, dans: Annales E. S. C. 37 (1982), p. 85; Kevin C. RoBBns, The Social Mechanisms of Urban Rebellion: a Case Study of Leadership in the 1614 Revolt at La Rochelle, dans: French Historical Studies $19 / 2$ (1995), p. 559-590.

${ }^{9}$ Constant, La Ligue (voir n. 6), p. 260.

${ }^{10}$ NiCOLAS, La rébellion française (voir n. 1), p. 10-11; Bernard CHEVALIER, Le pouvoir par le savoir: le renouvellement des élites urbaines en France au début de l'âge moderne (13501550), dans: Claude PETITFRÈRE (dir.), Construction, reproduction et représentation des patriciats urbains de l'Antiquité au XX' siècle, Tours 1999, p. 76.

${ }^{11}$ Robert DESCIMON, Qui étaient les Seize? Mythes et réalités de la Ligue parisienne, Paris 1983, p. 68.

${ }^{12}$ Barbara DiEfENDORF, The Catholic League: Social Crisis or Apocalypse Now?, dans: French Historical Studies 15/1 (1987), p. 332-344.

${ }^{13}$ Ibid., p. 337; Denis CROUZET, Les guerriers de Dieu. La violence au temps des troubles de religion (vers 1525-vers 1610), Seyssel 1990; LD., La représentation du temps à l'époque de la Ligue, dans: Revue Historique 548 (1983), p. 297-388. 
gueurs se déterminaient également en fonction de "visions du monde, de systèmes d'opinion, de niveaux d'intégration religieuse et idéologique « que l'on peut essayer de cerner en analysant l'expérience de ces villes ligueuses sous l'angle du comparatisme et de la culture politique, au sens propre du terme, de leurs dirigeants ${ }^{14}$.

Je tâcherai donc de montrer que, contrairement à une opinion communément répandue, la Ligue urbaine procèderait moins d'un réflexe réactionnaire de la part de cités sur le déclin que d'un puissant instinct de conservation, développé par des oligarchies anciennes et puissantes mais fragilisées, soucieuses de préserver leur héritage et de ménager l'avenir, ce qui transparaît nettement dans le comportement qu'elles ont observé durant cette période ${ }^{15}$. En dépit de quelques variantes locales et du cas particulier que constitue l'exemple parisien, on s'aperçoit, en effet, que les villes ligueuses se sont largement conformées à une sorte d'habitus commun, respectueux de leurs institutions, de leurs libertés et de l'ordre social. Même si des influences extérieures ont joué, en provenance de la capitale ou des princes, même si les contraintes de la guerre ont rendu inévitables quelques concessions voire quelques innovations, souvent limitées en l'occurrence, cette élite bourgeoise et catholique, placée à la tête des villes de l'Union, a clairement manifesté sa ferme volonté de sauvegarder coûte que coûte un »système urbain «, c'est-à-dire un ensemble de pratiques et de valeurs, à la fois fondatrices et fonctionnelles, garantes de la perpétuation de cette classe dirigeante mais aussi de la pérennité de la communauté citadine tout entière et que les contemporains ont cru menacées, dans la deuxième moitié du XVI ${ }^{e}$ siècle, par des évolutions politiques, sociales et religieuses échappant en grande partie à leur contrôle ${ }^{16}$.

Aujourd'hui, on ne croit plus guère à la "fable royaliste«, forgée de toutes pièces par les thuriféraires d'Henri IV et complaisamment entretenue ensuite par les historiens du $\mathrm{XIX}^{\mathrm{e}}$ siècle, rejetant la Ligue urbaine du côté de la lie du peuple, de la wcanaille endettée et des gibiers de potence dont la bassesse d'origine et la ruine matérielle et morale impliquaient la félonie ${ }^{17}$. On le doit à Henri Drouot qui a été le premier à s'intéresser au contenu social de la Sainte Union et à en fournir une théorie explicative. Selon lui, la Ligue serait le résultat d'un fort antagonisme entre deux bourgeoisies, celle de la judicature, monopolisant les offices des cours souveraines et cherchant à intégrer le second ordre et celle de la basoche, dans l'incapacité d'acquérir des charges

\footnotetext{
${ }^{14}$ DESCIMON, Qui étaient les Seize? (voir n. 11), p. 14.

${ }^{15}$ Fernand BRAUDEL, La Méditerranée et le monde méditerranéen à l'époque de Philippe II, Paris 1985, vol. II, p. 495; DESCIMON, Qui étaient les Seize? (voir n. 11), p. 12; CONSTANT, La Ligue (voir n. 6), p. 13.

${ }^{16}$ Yves BAREL, La ville médiévale. Système social, système urbain, Grenoble 1975.

${ }^{17}$ Descimon, Qui étaient les Seize? (voir n. 11), p. 12; ConSTANT, La Ligue (voir n. 6), p. 468; BARNAVI, DESCIMON, La Sainte Ligue (voir n. 6), p. 66-68.
} 
devenues trop onéreuses, du fait de la dégradation de la conjoncture économique et de leur confiscation par le jeu des résignations. C'est cette »bourgeoisie seconde«, contrariée dans son désir d'ascension sociale mais bien implantée dans les structures municipales qui, sous couvert de religion et de sauvegarde des libertés locales, aurait fourni ses forces vives à la Ligue bourguignonne. Ces avocats et ces procureurs, fils de marchands et gradués de l'université se seraient donc engagés dans l'Union, »non pour la servir par le chemin des grandes doctrines mais pour l'exploiter par instinct social«, dans une quête de puissance et de profit ${ }^{18}$. Voilà résumée une thèse qui est devenue par la suite un grand classique, au point que l'on parle aujourd'hui de "modèle Drouot« par rapport auquel les historiens de la Ligue ont été amenés à se positionner, soit en l'adoptant, soit en le rejetant ou bien encore en essayant de l'adapter ${ }^{19}$.

Ainsi, John Salmon affirme que les Seize parisiens étaient majoritairement des gens de loi, de rangs différents et dont la fragile alliance se serait rapidement défaite en raison de la "trahison« de la haute magistrature, modérée et proche de la noblesse et de la radicalisation des légistes subalternes, ayant vainement sombré dans l'activisme de rue et la violence afin de satisfaire leurs aspirations ${ }^{20}$. Toujours à propos de la Ligue parisienne, Élie Barnavi valide largement les assertions de Salmon mais aussi de Koenigsberger, en faisant de la Sainte Union »l'ancêtre des partis totalitaires modernes«, capable de recruter dans toutes les sphères de la société, doté d'une organisation bien structurée, à même de se substituer au gouvernement légal, porteur d'une idéologie très élaborée, usant, enfin, de la propagande et de la terreur comme outils de domination ${ }^{21}$. Cependant, la prise et l'exercice du pouvoir auraient rapidement conduit le "Parti de Dieu" à sa fragmentation interne et à son déclin. Son noyau dur, "constitué d'officiers de rang modeste et assimilés, d'avocats et de marchands, pour qui la Ligue était l'occasion rêvée de réaliser une impossible promotion«, aurait alors été rejeté dans le cercle vicieux de l'extrémisme et de l'isolement, tandis que ses membres les plus en vue socialement rejoignaient

${ }^{18}$ Henri Drouor, Mayenne et la Bourgogne (1587-1596). Contribution à l'histoire des provinces françaises pendant la Ligue, Thèse pour le doctorat ès lettres, Paris 1937, t. I, p. 33, $51,155-156$.

${ }^{19}$ Hilary J. BERnSTEN, The Bourgeoisie seconde, the Catholic League and Urban Society, dans: French History 17/4 (2004), p. 342-351.

${ }^{20}$ John Hersey SALMON, The Paris Sixteen, 1584-1594: The Social Analysis of a Revolutionary Movement, dans: ID., Renaissance and Revolution. Essays in the Intellectual and Social History of Early Modern France, Cambridge 1987, p. 235-266.

${ }^{21}$ Helmut Georg KoENIGSBERGER, The Organisation of Revolutionary Parties in France and the Netherlands during the Sixteenth Century, dans: ID., Estates and Revolutions. Essays in Early Modem European History, Londres 1971, p. 224-252; Élie BARNAVI, La Ligue parisienne (1585-1594): ancêtre des partis totalitaires modernes, dans: French Historical Studies 11 (1979), p. 29-57. 
le camp royaliste, par peur des conséquences sociales de cette révolution finalement manquée ${ }^{22}$. Car,

sur le plan social, non seulement personne ne songea à mettre en pratique le projet ligueur d'une noblesse de mérite, fondée sur l'intensité du sentiment religieux (Barnavi reprend ici le scritère d'intensité de la foi ‘ défini par Roland Mousnier comme fondement de la hiérarchie sociale que les ligueurs auraient aimé instaurer) mais encore ils retombèrent avec tous les sans offices dont ils avaient porté les espérances dans le marasme social qui fut le leur avant la Ligue $^{23}$.

À l'inverse de Barnavi, certains historiens anglo-saxons ne cautionnent pas du tout le »modèle Dijon-Paris ${ }^{24}$. Robert Harding ou Philip Benedict ne pensent pas, en effet, que la Ligue relève d'une "guerre sociale« entre deux bourgeoisies. Dans le cas d'Angers, Rennes et Nantes, Harding affirme que les ligueurs ne sont pas majoritairement issus de la pratique, mais des tribunaux royaux, et que les plus compromis ne sont pas forcément les plus âgés ou les gens du cru donc, a priori, les plus concernés par les affaires locales. L'auteur conclut par conséquent à l'absence d'explication sociale évidente, préférant mettre en avant l'incidence d'une sorte de wrévolution culturelle«, d'inspiration tridentine, axée sur une moralisation de l'Église, de l'État et de la société. À cet égard, il se situerait plutôt du côté de Denis Richet qui ne voyait pas la Ligue parisienne comme une »lutte de classes«, mais plutôt comme un

conflit aigu pour l'hégémonie culturelle et religieuse entre deux fractions inégalement pourvues de la bourgeoisie, entre les officiers des cours souveraines, soupçonnés sinon d'hérésie du moins de tiédeur catholique et le monde de la basoche, ces procureurs, ces huissiers, ces notaires, ces petits avocats, ces sergents, liés à la boutique et à l'échoppe, frustrés dans leurs ambitions de promotion sociale, défenseurs jaloux d'une orthodoxie volontiers niveleuse ${ }^{25}$.

Pour Philipp Benedict également, la Ligue rouennaise n'est pas une révolte d'avocats et de marchands aigris; elle ne correspond pas non plus à la victoire de ce qu'il appelle des »outs«, écartés du pouvoir par des »ins«, l'ayant confisqué. Il s'agit plutôt d'une lutte entre sins « opposant, au sein du parlement, les anciens et les nouveaux pourvus mais aussi les officiers plus matures, ayant fait l'expérience du péril huguenot dans les années 1560 et les plus jeunes qui tendraient plutôt du côté d'une monarchie forte, seule capable de mettre un

${ }^{22}$ ID., Le parti de Dieu. Étude sociale et politique des chefs de la Ligue parisienne (15851594), Bruxelles, Louvain 1980, p. 51-52.

${ }^{23}$ Roland MOUSNIER, Les hiérarchies sociales de 1450 à nos jours, Paris 1969, p. 51-54; BARNAVI, Le parti de Dieu (voir n. 22), p. 258-261.

${ }^{24}$ Robert HARDING, Revolution and Reform in the Holy League: Angers, Rennes, Nantes, dans: The Journal of Modern History 53 (1981), p. 392.

${ }^{25}$ Denis RICHET, Aspects socioculturels des conflits religieux à Paris dans la seconde moitié du XVI ${ }^{e}$ siècle, dans: Annales E. S. C. 32 (1977), p. 775-780. 
terme à la guerre civile ${ }^{26}$. Plus récemment, Mack Pee Holt est allé jusqu'à récuser l'analyse de Drouot en révélant, chiffres à l'appui, que, pendant et après la Ligue, les membres de la »bourgeoisie seconde« dijonnaise n'ont nullement accru leur emprise sur l'hôtel de ville qui est resté aux mains des marchands et des autres bourgeois. De son point de vue, les divisions de la Ligue sont donc davantage le fait de choix politiques difficiles et de toute une série de facteurs plus complexes que les tensions existant entre les deux bourgeoisies ${ }^{27}$. De même, Sara Beam démontre, à l'aide des archives du parlement de Paris, que la plupart des procureurs et des clercs de justice ne soutenaient pas l'entreprise séditieuse des Seize. Au contraire, ceux-ci sont restés dans la capitale afin de ne pas perdre leur gagne-pain au sein d'un parlement croupion, déployant même de grands efforts afin de demeurer dans les bonnes grâces des magistrats royaux ${ }^{28}$.

Entre ces deux positions nettement tranchées, certains historiens de la Ligue refusent de choisir. Pour Wolfgang Kaiser, par exemple, la Ligue marseillaise relève d'abord de la lutte de factions, »menée par des groupes concurrents de l'élite politique, désireux de s'emparer du pouvoir et d'accéder aux commandes de la vie économique, d'atteindre ou de briser une hégémonie«. Cela dit, il ne rejette pas complètement la thèse de Drouot qu'il module en disant que la faction de Casaulx (c'est-à-dire des ligueurs radicaux) peut être considérée comme "l'expression d'une bourgeoisie seconde avec une coloration marseillaise spécifique, qui se dresserait contre les tendances de l'aristocratie marchande à se couper de l'ensemble de la communauté bourgeoise ${ }^{29}$.

S'agissant de la Ligue limougeaude, Michel Cassan propose également "d'envisager la thèse de Drouot dans une acception élargie«. Si, dans cette ville, le clivage entre basoche et magistrature ne joue pas, il existerait, en revanche, entre "deux strates très différentes du monde des officiers de finances«, c'est-à-dire entre les gens du Bureau de Finances et ceux de l'Élection ${ }^{30}$. Mais là n'est pas l'essentiel, qui gît plutôt dans le "refus d'une marginalisation« de la part de notables soucieux d'empêcher l'absorption du système de la Ville par celui de l'État, c'est-à-dire

un déplacement du pouvoir (du consulat vers le présidial) qui amplifierait les forces de recomposition sociale déjà à l'œuvre en leur sein, privant certains de responsabilités civiques

${ }^{26}$ Philip BENEDICT, Rouen during the Wars of Religion, Cambridge 1981, p. 182-185.

${ }^{27}$ Mack P. HoLT, The League in Burgundy: A Bourgeoisie seconde?, dans: French History $17 / 4$ (2004), p. 352-366.

${ }^{28}$ Sara BEAM, The Basoche and the Bourgeoisie seconde: Careerists at the Parlement of Paris during the League, dans: French History 17/4 (2004), p. 367-387.

${ }^{29}$ Wolfgang KAISER, Marseille au temps des troubles. Morphologie sociale et luttes de factions (1559-1596), Paris 1992, p. 42, 166, 341-342.

${ }^{30}$ Michel CASSAN, Le temps des guerres de religion. Le cas du Limousin (vers 1530vers 1630), Paris 1996, p. 275. 
compensatrices et débouchant sur la formation d'une nouvelle hiérarchie, fondée uniquement sur la possession des offices royaux et non sur les critères traditionnels, tels que l'ancienneté de la famille, le prestige de la parentèle ${ }^{31}$.

Cette hypothèse s'inspire en grande partie de la thèse que Robert Descimon a élaborée à propos de la Ligue parisienne. Ce dernier soutient, en effet, que »la guerre des deux bourgeoisies n'est pas le conflit premier « et qu'en réalité, "l'antagonisme principal dresse la Ville contre l'État ${ }^{32}$. Ainsi, au-delà du "clientélisme intéressé des oligarques, de la lutte de corps qui mobilisait une partie de la basoche contre la robe, de l'effort des marchands pour conserver fortune et notabilité«, les ligueurs parisiens poursuivaient, selon lui, le même projet politique d'unité bourgeoise, défendaient une conception spécifique de la ville comme »corps commun« et ce, en réponse aux »puissants facteurs de dissolution« que représentaient les clientèles nobiliaires, le service de l’État mais aussi le »collapsus du premier capitalisme marchand« et le schisme religieux ${ }^{33}$.

Bien qu'elle ne puisse être transposée telle quelle à toutes les villes ligueuses, cette analyse a profondément influencé la façon de percevoir leur engagement. Non seulement, elle a souligné la nécessité de le resituer dans un tissu social complexe mais elle a également attiré l'attention sur l'adhésion des bourgeois ligueurs à une culture originale, proprement civique, sur leurs aspirations à défendre une certaine forme de républicanismer à propos de laquelle, cependant, il ne faut pas se tromper en la réduisant à une simple contestation de l'ordre monarchique ou à la volonté de confier tout ou partie de la souveraineté au "peuple $\ll^{34}$.

De fait, parmi les ıfables` que les contemporains ont bâties à propos de la Ligue urbaine, on trouve celle d'une pseudo tentation républicaine de la part de cités qui auraient nourri le dessein de s'affranchir de l'autorité monarchique pour se gouverner seules, comme autant de petits États autonomes, à la manière de certaines villes italiennes, germaniques ou néerlandaises ${ }^{35}$. Ainsi, début janvier 1589, dans une lettre à Henri III, le duc de Longueville, gouverneur de Picardie, parle de république imaginaire pour qualifier le comportement séditieux de la municipalité amiénoise ${ }^{36}$. D'autres chefs royalistes voire des princes ligueurs utilisent la même expression pour stigmatiser le comporte-

${ }^{31}$ ID., Mobilité sociale et conflits religieux: l'exemple limousin (1530-1630), dans: La dynamique sociale dans l'Europe du Nord-Ouest (XVI'-XVII siècles), Paris 1987, p. 82-83.

${ }^{32}$ DESCIMON, Qui étaient les Seize? (voir n. 11), p. 25.

${ }^{33}$ Ibid., p. 26, 295-296.

34 Éric GoJosso, Le concept de république en France (XVI-XVII ${ }^{\mathrm{e}}$ siècles), Aix-enProvence 1998, p. 172-178.

${ }^{35}$ Yves DURAND, Les républiques au temps des monarchies, Paris 1973; Gojosso, Le concept de république (voir n. 34), p. 217-218.

${ }^{36}$ Bibliothèque nationale, Département des manuscrits, ms. fr 3363, fol. 225-228. 
ment de corps de villes refusant de se plier à leur volonté et revendiquant leur indépendance ${ }^{37}$.

Or, il faut ici se garder de tout contresens. Il est possible que certaines villes, tournées vers la mer et le grand commerce ou sises dans des provinces périphériques, tardivement rattachées au domaine de la couronne aient pu caresser le rêve d'une véritable émancipation et agir en conséquence, à l'instar de Marseille ou de Saint-Malo ${ }^{38}$. Cela dit, elles ne sont pas la norme et leur tentative a fait long feu puisque non seulement, elles ont fini par se rallier à Henri IV comme toutes les autres, mais elles n'ont pas non plus été reconnues dans le concert des nations européennes de l'époque, à l'inverse des Provinces-Unies, par exemple ${ }^{39}$.

Néanmoins, il est vrai que certains aspects du comportement des grandes villes ligueuses seraient susceptibles d'étayer l'accusation qu'on a formulée à leur égard, sachant que leur participation à l'Union s'est traduite, dans la plupart des cas, par une soustraction d'obédience, à la fois effective et symbolique, envers le monarque, par l'usurpation de droits purement régaliens et par la tentative d'étendre leur souveraineté aux villes voisines et sur le plat pays, au détriment de la sienne.

À Amiens, par exemple, au lendemain des événements de Blois, l'échevinage brûle les lettres du roi sans les lire, refuse d'ouvrir les portes de la ville à ses émissaires et fait emprisonner ses représentants et leur suite. De même, tout comme à Marseille où on cessa de prier pour Henri III et de le citer dans les cris publics, la cité phocéenne préférant se placer sous la souveraineté du Christ, dans la capitale picarde, on déclare ne vouloir obéir désormais qu'au roi céleste et immortel, le roi terrien s'étant rendu infidèle à Dieu et à son peuple. C'est pourquoi, quelques mois plus tard, à l'occasion d'une fête civique importante, ce n'est pas au nom du roi, comme il en avait coutume, que le corps de ville offre un cierge à la Vierge mais au nom de la couronne ${ }^{40}$. Enfin, avant même la promulgation du décret de la Sorbonne déliant les Français de leur serment de fidélité envers Henri III, dans une déclaration imprimée, l'échevinage amiénois invite solennellement les bons catholiques à s'opposer de toutes leurs forces aux pernicieulx desseins et machinations des ennemis de Dieu et de son Eglise, au premier rang desquels figure le dernier Valois ${ }^{41}$.

\footnotetext{
${ }^{37}$ Yves DURAND, Les républiques urbaines en France à la fin du XVI siècle, dans: Annales de la société d'histoire et d'archéologie de l'arrondissement de Saint-Malo (1990), p. 224 225, 238-239.

${ }^{38}$ Ibid., p. 225, 231; BRAUDEL, La Méditerranée (voir n. 15), p. 495.

${ }^{39}$ DURAND, Les républiques urbaines (voir n. 37), p. 243: l'auteur parle de mrépubliques avortées«.

${ }^{40}$ KAISER, Marseille au temps des troubles (voir n. 29), p. 287-288; Olivia CARPI, Une république imaginaire. Amiens pendant les troubles de religion (1559-1597), Paris 2005, p. 132-133.

${ }^{41}$ Bibliothèque nationale de France, Département des imprimés, $\mathrm{Lb}^{34} 630$.
} 
Parallèlement, à Amiens, Lyon, Dijon, Rouen, Toulouse ou Marseille, on n'hésite pas, au nom des nécessités de la guerre, à battre monnaie, à lever de nouveaux impôts sur les habitants de la ville et des alentours ou à détourner le produit de ceux que le roi percevait dans le pays ${ }^{42}$. À Amiens, notamment, le corps de ville ligueur, de sa seule autorité, prononce la confiscation des recettes fiscales de la province, obligeant, sous peine de représailles, tous les receveurs à venir vider leurs mains dans une Recette générale de l'Union, gérée par un ligueur notoire qui s'arroge les fonctions normalement exercées par les officiers du Bureau de finances qui, s'ils ne sont pas arrêtés et emprisonnés ou privés de leurs gages, se voient dépouillés arbitrairement de leur charge au profit d'officiers subalternes plus "zélés « ${ }^{43}$. On assiste donc bien à une tentative de "municipalisation de l'argent public« qui se révèle cependant une expérience peu durable dans la mesure où, dénués de l'expertise mais aussi de l'autorité nécessaires, les cadres ligueurs amiénois finissent par réintégrer les officiers supérieurs de finance, soumis toutefois à une étroite surveillance afin qu'ils ne soient pas tentés de travailler contre les intérêts de l'Union ${ }^{44}$.

Comble de la subversion, cet argent est utilisé pour payer les troupes que les villes rebelles opposent aux forces royalistes chargées de les ramener à l'obéissance. Estimant insuffisantes leurs fortifications et leurs milices et refusant de tomber sous la coupe d'une garnison, les villes ligueuses recrutent effectivement des capitaines et des compagnies de gens d'armes, de pied ou de cheval, qu'elles rétribuent sur les deniers communs et dont les municipalités revendiquent en contrepartie le commandement, fixant à la fois le montant des soldes, les règles de partage du butin et les objectifs stratégiques, tout en nommant certains de leurs membres pour veiller à l'application de leurs décisions ${ }^{45}$.

Certes, cela vaut surtout pour les villes qui n'ont pas été placées sous la tutelle de gouverneurs militaires, sachant néanmoins que ceux-ci s'absentent souvent pour la conduite des opérations et que ce sont les municipalités qui tiennent les cordons de la bourse, ce qui leur confère une réelle marge de manœuvre. En fait, ce rejet d'une quelconque inféodation de la part des dirigeants bourgeois s'applique à l'ensemble des représentants du second ordre, étant donné que si les villes catholiques insurgées ne pensent pas pouvoir se passer de protecteurs princiers et de cadres militaires recrutés dans la gentilhommerie, il ne saurait être question de laisser ces derniers se comporter en

42 Peter Marc ASCOLI, French Provincial Cities and the Catholic League, dans: Occasional Papers of the American Society for Reformation Research 1 (1997), p. 15-40, à la p. 28.

${ }^{43}$ CARPI, Une république imaginaire (voir n. 40), p. 185-186.

44 Pierre-Jean SoURIAC, Comprendre une société confrontée à la guerre civile: le Midi toulousain entre 1562 et 1596, dans: Histoire, Economie, Société 2 (2004), p. 77, 86; CARPI, Une république imaginaire (voir n. 40), p. 186.

${ }^{45}$ CASSAN, Le temps des guerres de religion (voir n. 30), p. 168-170; SOURIAC, Comprendre une société (voir n. 44), p. 77-88; CARPI, Une république imaginaire (voir n. 40), p. 170172. 
maîtres impérieux, quitte à entrer en conflit ouvert avec eux ${ }^{46}$. Dès lors, Mayenne, Aumale, Nemours, Mercœur et leurs sbires ont beau menacer de tout mettre à feu et à sang pour être obéis, il leur faut tempérer leurs ardeurs et leurs appétits, au risque de s'aliéner leurs principaux soutiens, à la fois politiques et financiers. En témoigne, par exemple, l'insistance dont le duc de Mayenne a du faire preuve pour convaincre les villes de l'Union d'envoyer des députés aux États de la Ligue qui, indépendamment des périls du voyage, ne suscitaient guère l'enthousiasme des bourgeois, peu désireux d'être considérés comme de simples pions dans le jeu des Grands, surtout quand ceux-ci ne leur apportaient pas l'aide qu'ils avaient promise ${ }^{47}$. C'est d'ailleurs ce qui explique que Mayenne et les autres princes catholiques aient tant milité en faveur de la création de conseils de l'Union dans les cités concernées, espérant de cette manière faire pièce aux municipalités trop rétives voire s'en servir comme conseils de gouvernement dans les provinces placées sous leur domination ${ }^{48}$.

Le même genre de rapports, "obligés mais toujours méfiants«, s'instaure également entre les villes ligueuses qui professent la solidarité, tout en se refusant à subir la domination de la capitale du royaume ou de l'une de leurs semblables $^{49}$. Ainsi, dès leur adhésion au mouvement ligueur, Paris et les grandes villes comme Amiens, Rouen ou Toulouse ont cherché, par la persuasion ou la contrainte, exercée sous forme de blocus commercial ou d'ostracisme, à entraîner leurs voisines à leur suite ${ }^{50}$. Or, si d'emblée des liens étroits se nouent entre villes ligueuses, à la faveur de la conclusion d'alliances sanctionnées par des serments solennels et débouchant sur l'adoption de revendications communes ou sur une entraide financière et militaire, les velléités hégémoniques des plus importantes d'entre elles achoppent sur des obstacles objectifs tels que les difficultés de communication ou de financement d'actions de grande ampleur, l'absence de relais institutionnels et, surtout, sur l'esprit de clocher très vivace de bonnes villes jalouses de leurs libertés, souvent difficilement acquises et soucieuses avant tout de leur propre sauvegarde ${ }^{51}$.

${ }^{46}$ DURAND, Les républiques urbaines (voir n. 37), p. 216, 237-238.

${ }^{47}$ Ibid., p. 216, 237-238; CARPI, Une république imaginaire (voir n. 40), p. 173-174.

${ }^{48}$ Henri DroUOT, Les conseils provinciaux de la Sainte Union (1589-1595). Notes et questions, dans: Annales du Midi 45 (1953), p. 415-433.

${ }^{49}$ DURAND, Les républiques urbaines (voir n. 37), p. 235.

${ }^{50}$ Ibid., p. 227, 235; Ariane BoLTANSKI, Les solidarités entre les villes ligueuses du Bassin parisien, Mémoire de maîtrise sous la direction de Nicole Lemaître, université de Paris I, 1991; Marck GrEENGRASS, The Sainte Union in the Provinces: The Case of Toulouse, dans: Sixteenth Century Journal 14 (1983), p. 488-489; CARPI, Une république imaginaire (voir $n$. 40), p. 162-156; EAD., Entre solidarité militaire et velléités fédératives: le réseau des villes ligueuses de Picardie, dans: Philippe BOULANGER, Philippe Nrvet (dir.), La géographie militaire de la Picardie, Amiens 2006.

${ }^{51}$ Élie BARNAvI, Centralisation ou fédéralisme? Les relations entre Paris et les villes à l'époque de la Ligue (1585-1594), dans: Revue Historique 526/2 (1978), p. 339-340; An- 
On en veut pour preuve l'échec de la tentative d'instauration d'États provinciaux à Dijon, Lyon, Nantes, Poitiers, Rouen, Troyes ou Amiens. Dans tous les cas de figure, malgré le soutien du Conseil parisien et des princes, ces assemblées ne sont jamais allées plus loin que le stade de projet ou de simulacre, du fait de l'absence de tradition représentative dans la plupart de ces provinces, dénuées d'États ou précocement rattachées au domaine de la couronne, mais aussi de la force de l'héritage communal dans les villes du nord de la Loire et, dans tous les cas, d'une sorte d'égoïsme sacré qui a conduit les villes ligueuses à refuser de faire bourse commune et, plus tard, à renouer des relations commerciales avec celles du camp adverse ou à entamer des négociations séparées, lorsque la Sainte Union est entrée en décadence ${ }^{52}$.

Que les villes ligueuses aient fait l'expérience d'une grande autonomie est donc incontestable, mais il importe de replacer cette politique dans son contexte. Tout d'abord, il n'est pas douteux que certaines mesures extrêmes ont été prises sous le coup du traumatisme occasionné aux consciences catholiques par l'assassinat des Guises et la dissolution brutale des états généraux qui constituait de la part du roi une irréparable violation de la liberté et de la foy publicques dont il était censé être le garant en second lieu ${ }^{53}$. D'autres décisions, attentatoires à la souveraineté monarchique ne relèvent que de l'expédient, imposé aux édiles ligueurs par l'urgence et la nécessité que généraient leurs difficultés budgétaires et la menace d'une entreprise militaire. D'ailleurs, à chaque initiative de ce genre, on a fait en sorte de respecter les formes légales en sollicitant l'aval des cours souveraines, il est vrai, largement gagnées à la Ligue ou en s'autorisant du duc de Mayenne, lieutenant général de la couronne voire du roi légitime à venir ou du pape ${ }^{54}$. Enfin, certains gestes peuvent être interprétés, soit comme la manifestation d'un esprit de revanche par rapport à la politique récente de la monarchie, jugée attentatoire aux immunités citadines, en particulier dans le domaine financier, soit comme la traduction d'authentiques aspirations réformatrices de la part de ces dirigeants citadins, désireux de substituer au régime administratif en place celui, plus respectueux des libertés locales, auquel ils croyaient encore.

L'arbre ne doit donc pas cacher la forêt. Ce n'est pas tellement dans ces mesures somme toute exceptionnelles que se laisse apercevoir le système de représentations à l'œuvre dans cette insurrection bourgeoise mais, paradoxalement, dans une attitude très conformiste, caractérisée par une réelle modération, la recherche d'une certaine unanimité et un conservatisme sourcil-

nette FINLEY CROSWHITE, Confederate and Rivals: Picard Urban Alliances during the Catholic League (1588-1594), dans: Annales canadiennes d'histoire 31 (1996), p. 359-376.

${ }^{52}$ ASCOLI, French Provincial Cities (voir n. 42), p. 24-26.

${ }^{53}$ WILKINSON, Homicides royaux (voir n. 7), p. 129-153; CONSTANT, La Ligue (voir n. 6), p. 213-219; Archives communales de la ville d'Amiens antérieures à 1790, AA 121, pièce n' 82.

${ }^{54}$ DURAND, Les républiques urbaines (voir n. 37), p. 225, 239. 
leux. De fait, si surtout dans les premiers temps de la »Ligue offensive«, il a fallu se résoudre à quelques compositions, superficielles et temporaires, les bourgeois ligueurs restent globalement fidèles à un mode de gouvernement dont ils sont à la fois les dépositaires et les principaux bénéficiaires. C'est pourquoi, on n'assiste pas, dans la plupart des cas, à une sorte de sterreur ligueuse«, ni à une véritable "poussée démocratique» et encore moins à une subversion des institutions établies qui frappent au contraire par leur stabilité.

Pour commencer, nonobstant certains événements isolés comme l'assassinat du président Brisson à Paris ou celui de Duranti à Toulouse, ou encore quelques rixes sanglantes lors du passage à l'Union comme au Mans, celle-ci n'est pas synonyme d'un déchaînement de la violence qui a plutôt tendance à refluer $^{55}$. Cela vaut en particulier pour les réformés qui ne sont pas victimes d'une nouvelle "pulsion massacrante« mais traités comme les autres adversaires de l'Union ${ }^{56}$. Ceulx du party contraire, mêlant royalistes avérés ou putatifs, sont en effet arrêtés, emprisonnés ou assignés à résidence, privés de leurs charges, exclus de toutes les manifestations publiques, mis à rançon et parfois momentanément bannis, lorsque la ville se sent menacée d'une "surprise « de la part des royaux dont ces derniers pourraient se rendre complices. Or, on constate que ces dispositions ont surtout pour objet de protéger ceux du parti contraire d'éventuelles agressions d'un peuple à l'émotivité redoublée par un contexte éprouvant, mais aussi afin de les empêcher de nuire à l'Union, par la propagande ou la conspiration ${ }^{57}$. D'ailleurs, en échange de fortes cautions et de la promesse de se tenir tranquilles, la plupart de ces prisonniers royalistes sont rapidement libérés de leurs geôles et voient leur traitement sensiblement adouci, surtout lorsqu'ils sont issus de la même famille que les ligueurs.

Car l'obsession des édiles ligueurs reste plus que jamais la préservation de la paix publique, indispensable au maintien de leur autorité mais aussi à la sûreté de la ville et à sa survie économique, déjà fortement compromises. C'est la raison pour laquelle les forces de maintien de l'ordre sont décuplées, la population placée sous étroite surveillance, grâce notamment à des bourgeois faisant office d'espions pour le compte de la municipalité et tout écart de conduite sévèrement réprimé, même lorsqu'il se justifie par une itension d'agression ${ }^{58}$. Parallèlement, les municipalités ligueuses s'ingénient à susciter ou à entretenir la cohésion de la communauté citadine. Cela passe, pour commencer, par la volonté de réduire coûte que coûte la fracture confessionnelle,

${ }^{55}$ CONSTANT, La Ligue (voir n. 6).

${ }^{56}$ Denis CrouzET, Le règne de Henri III et la violence collective, dans: Robert SAUZET (dir.), Henri III et son temps, Paris 1992, p. 214-223.

${ }^{57}$ CASSAN, Le temps des guerres de religion (voir n. 30), p. 269-271; Laurent BOURQUIN, Les nobles, la ville et le roi. L'autorité nobiliaire en Anjou pendant les guerres de religion, Paris 2001, p. 148-158; CARPI, Une république imaginaire (voir n. 40), p. 150-152.

${ }^{58}$ Denis CROuzet, Les Guerriers de Dieu. La violence au temps des guerres de religion, Seyssel 1990, p. 264; CARPI, Une république imaginaire (voir n. 40), p. 153-155. 
synonyme d'une pacification des relations entre catholiques et protestants mais aussi d'un refus de toute forme de tolérance, les réformés repentants étant aisément réintégrés dans la communauté alors que les plus obstinés se voient condamnés à une véritable mort sociale ${ }^{59}$. On observe également la recherche d'un certain consensus sur la teneur de la politique municipale, à coups de prédications enflammées, d'habile propagande imprimée et de réunions d'assemblées d'habitants, souvent plus fréquentes et plus ouvertes qu'auparavant mais largement cantonnées dans une fonction de ratification des décisions prises à l'hôtel de ville ${ }^{60}$. Poursuit la même finalité de réconciliation des habitants avec eux-mêmes et avec Dieu la mise en cuvre de toute une gamme de srites d'unité ${ }^{61}$, à la croisée du politique et du religieux, tels que la célébration en grande pompe des funérailles des princes martyrs, la prestation récurrente et solennelle de serments, rendus obligatoires sous peine de bannissement ou l'organisation de grandes processions expiatoires et de prières publiques ${ }^{62}$.

Élus pour faire respecter l'honneur de Dieu et de son Église et pour œuvrer au bien et repos de tous leurs concitoyens, les magistrats municipaux le sont aussi pour assurer l'intégrité et la pérennité d'un système de gouvernement, parfois pluriséculaire et permettant la reproduction d'une oligarchie qui y trouve à la fois pouvoir, honneur et profit, en échange de son investissement au service du bien commun ${ }^{63}$. Dès lors, on ne s'étonnera pas de constater qu'à de rares exceptions près, telles que le prolongement du mandat d'un maire ou le remplacement d'échevins suspects par des plus zélés, parfois issus de classes socioprofessionnelles tenues jusque là à l'écart du jeu municipal, globalement, les règles de recrutement et de fonctionnement des corps de villes ne sont ni modifiées ni renversées pendant l'épisode ligueur ${ }^{64}$. En fait, dans les villes qui n'ont pas été victimes d'un coup de force et dotées d'une tradition municipale solide, une sorte d'alternance entre ligueurs et royalistes a été faci-

59 David NICHOLLS, Protestants, Catholics and Magistrats in Tours, 1562-72: The Making of a Catholic City, dans: French History 8/1 (1994), p. 14-33; Barbara B. DIEFENDORF, Beneath the Cross. Catholics and Huguenots in XVI ${ }^{\text {th }}$ Century Paris, Oxford 1991; CARPI, Une république imaginaire (voir n. 40), p. 80-89.

${ }^{60}$ Philippe PAPIN, Duplicité et traîtrise: l'image des Politiques durant la Ligue, dans: Revue d'histoire moderne et contemporaine 38 (1991), p. 3-21; David A. BELL, Unmasking a King: The Political Uses of Popular Literature under the French Catholic League (1588-89), dans: Sixteenth Century Journal 20/3 (1989), p. 371-386; DURAND, Les républiques urbaines (voir n. 37), p. 223, 232.

${ }^{6 l}$ Denis Crouzet, La représentation du temps à l'époque de la Ligue, dans: Revue historique 270/2 (1983), p. 370-371.

${ }^{62}$ Ibid., p. 224-226; Ann W. RAMSEY, Liturgy, Politics and Salvation: The Catholic League in Paris and the Nature of Catholic Reform (1540-1630), Rochester 1999.

${ }^{63}$ CARPI, Une république imaginaire (voir n. 40), p. 63; BLICKLE (dir.), Résistance, représentation et communauté (voir n. 4), p. 258-271.

${ }^{64}$ Penny RoBerts, A City in Conflict. Troyes during the French Wars of Religion, Manchester 1996, p. 176. 
litée par le jeu des factions et l'attachement de l'élite bourgeoise aux constitutions locales, arrachées de haute lutte au temps de l'émancipation communale ou plus récemment obtenues d'un seigneur ou du monarque ${ }^{65}$. De même, les conseils de l'Union, ouverts aux représentants des trois ordres et qui se sont multipliés dans les villes catholiques ne se sont jamais substitués aux organes municipaux dont ils ne sont restés que de simples auxiliaires jusqu'à leur mise en sommeil ou leur disparition précoce, fin $1589^{66}$.

Enfin, cette défense achamée de leurs acquis s'exprime clairement dans les conditions que les villes ligueuses mettent à leur ralliement à Henri IV, à qui elles ne réclament rien d'autre qu'une amnistie générale, la prohibition du culte réformé dans leurs murs et la conservation des droits et prérogatives dont elles jouissaient jusque là ${ }^{67}$.

Ainsi, le comportement des villes ligueuses ne doit pas seulement se lire à la lumière du conflit confessionnel ou des tensions qui traversaient les élites bourgeoises en voie de recomposition, sous l'effet du développement de la vénalité des offices et d'une roligarchisation croissante des gouvernements citadins. Leur expérience spécifique de la révolte s'éclaire aussi par une connaissance approfondie du système politique qui s'est développé depuis le Moyen Âge dans la plupart des bonnes villes du royaume, compendium de normes et de discours, de droits et d'usages, auquel les membres de l'élite bourgeoise étaient viscéralement attachés, non seulement parce qu'il leur donnait la possibilité de se maintenir aux commandes de la ville parfois plusieurs générations de suite, avec toutes les gratifications honorifiques et matérielles que cela impliquait, mais aussi parce qu'il formait l'une des composantes essentielles d'une identité citadine, hautement revendiquée face au roi, à la noblesse et au plat pays ${ }^{68}$.

Dès lors, bien que périlleux, l'engagement ligueur n'a rien d'une aberration. Il se révèle au contraire parfaitement cohérent avec une méthode de gouvernement et un système de représentations qui plaçaient l'élite bourgeoise dans une position à la fois valorisante et contraignante, en situation d'interface entre le monarque et une communauté dont l'institution municipale était à la fois l'émanation, même indirecte, l'instrument et le garant. Or, face à une crise

${ }^{65}$ Jean-Marie CONSTANT, Pouvoir municipal et patriciat dans une ville de l'Ouest de Louis XI à la Révolution: le Mans, dans: PEMTFRERE (dir.), Construction, reproduction et représentation des patriciats urbains (voir n. 10), p. 297-307; ID., La Ligue (voir n. 6), p. 299-306; BOURQUIN, Les nobles, la ville et le roi (voir n. 57), p. 137.

${ }^{66}$ DROUOT, Les conseils provinciaux (voir n. 48), p. 415-433; CARPI, Une république imaginaire (voir n. 40), p. 156-158.

${ }^{67}$ Michel CASSAN, La réduction des villes ligueuses à l'obéissance, dans: Nouvelle Revue du XVI' siècle 22/1 (2004), p. 159-174.

${ }^{68}$ Bernard Chevalier, Les bonnes villes de France, Paris 1982; Hilary J. Bernstern, Between Crown and Community. Politics and Civic Culture in Sixteenth Century Poitiers, Ithaca 2004; CARPI, Une république imaginaire (voir n. 40). 
multiforme, alliant rupture de l'unité de foi, exigences croissantes de la royauté, spécialement dans le domaine financier, dégradation de la conjoncture économique et accumulation de calamités, la classe dirigeante des bonnes villes catholiques a vu dans la Ligue le moyen de sauvegarder ce qu'elle s'était ingéniée à édifier et entretenir au fil des années, bref, de poursuivre l'idéal républicain qui sous-motivait son action c'est-à-dire une certaine conception de la chose publique, se caractérisant par la pratique d'un "catholicisme corporatif ${ }^{69}$, le gouvernement éclairé de la sanior pars bourgeoise et l'entretien avec la monarchie d'une relation d'essence contractuelle, fondée sur un principe de réciprocité ${ }^{70}$.

En initiant ou en encadrant la révolte ligueuse, les membres de l'élite bourgeoise ne faisaient donc pas que revendiquer une certaine supériorité sociale, même si ces considérations n'étaient pas étrangères à leur démarche, loin s'en faut. Plus qu'un statut, c'est une identité qu'ils défendaient avec acharnement, une façon d'être et de penser qui se donnait à voir aussi bien dans la pierre que dans l'étoffe, dans le discours que dans les gestes, cérémoniels ou quotidiens, et qui n'était pas sans rencontrer un certain consensus au sein de la population citadine, en dépit des tiraillements occasionnés par le schisme religieux et les "malheurs des temps«. Tributaires d'une vision organiciste du monde, les bourgeois ligueurs prétendaient en effet rester fidèles à la fonction qu'ils pensaient devoir remplir au sein du corps citadin, au service de sa survie à la fois temporelle et spirituelle ${ }^{71}$. Partant, loin de vouloir restaurer un hypothétique âge d'or, ils souhaitaient seulement préserver un régime politique et social ayant atteint son apogée dans la première moitié du siècle, mais durement ébranlé dans la deuxième par une crise d'une gravité inusitée ${ }^{72}$.

Bien que leur vécu conserve une part d'irréductibilité, les villes ligueuses partagent donc une véritable culture, une certaine forme de srépublicanisme civique«, d'autant plus vivace que sévèrement malmenée durant la deuxième moitié du XVI siècle $^{73}$. Aussi la Ligue ne doit-elle pas être vue comme le sur-

${ }^{69}$ Robert DESCIMON, Le corps de ville et le système cérémoniel parisien au début de l'âge moderne, dans: Marc BOONE, Maarten PRAK (dir.), Statuts individuels, statuts corporatifs et statuts judiciaires dans les villes européennes (Moyen Âge et Temps modernes), Louvain, Apeldoorn 1996, p. 73-128.

${ }^{70}$ Philippe GuIGNET, Le pouvoir dans la ville au XVIII ${ }^{e}$ siècle, Paris 1990, p. 79-91; Robert A. SCHNEDER, Public Life in Toulouse. From Municipal Republic to Cosmopolitan City, Ithaca, Londres 1989, p. 59-81; CARPI, Une république imaginaire (voir n. 40), p. 34-37.

${ }^{7}$ NiCOLAS, La rébellion française (voir n. 1), p. 13: "le recours collectif à la violence s'inscrit toujours dans le système de représentations par lequel la communauté désigne sa place dans l'ordre du monde, ces images mentales qui se traduisent en actes renvoient donc à du sens et le conflit est à lire non comme pur désordre mais comme révélateur d'une cohérence autre

${ }_{72}^{72}$ Stéphane GAL, Grenoble au temps de la Ligue, Grenoble 2000, p. 567.

${ }^{73}$ Heinz SCHILLING, Religion, Political Culture and the Emergence of Early Modern Society, Leyde, New York, Cologne 1992, p. 3-59. 
saut désespéré de communes moribondes, mais comme la traduction d'un idéal politique éminemment mobilisateur et d'une réelle modernité, qui ne se réduit pas nécessairement aux efforts de sécularisation et de centralisation qu'affichait la monarchie. En cela, les villes ligueuses sont bien des »républiques imaginaires«, le creuset où l'on a élaboré et expérimenté un modèle de gouvernement offrant une alternative à celui que l'État royal s'efforçait laborieusement de faire prévaloir, en vertu de logiques qui, quoiqu'on en dise, n'avaient rien d'irréversible ${ }^{74}$.

${ }^{74}$ BLICKLE (dir.), Résistance, représentation et communauté (voir n. 4), p. 132-133, 252, 272, 294-295. 
